\title{
Insertion of a tracheoesophageal speech valve: a novel approach in a difficult situation
}

\author{
C P YIANNAKIS, R B TOWNSLEY, I G SMILLIE, G L PICOZZI
}

Department of Otolaryngology, Monklands District General Hospital, Airdrie, Scotland, UK

\begin{abstract}
Background: Functional voice rehabilitation is becoming increasingly important following total laryngectomy and pharyngolaryngectomy. Tracheoesophageal voice via a shunt valve is currently regarded as the 'gold standard' for voice rehabilitation. Traditional techniques usually allow for the replacement of valves in the out-patient setting; however, patient factors such as altered anatomy may occasionally prevent this.

Objective: This paper describes a novel approach for speech valve insertion that is safe, quick and cost-effective, and which uses equipment commonly available in ENT wards and the operating theatre.
\end{abstract}

Key words: Laryngectomy; Pharyngectomy; Speech, Laryngeal; Speech, Esophageal; Speech Therapy; Voice Prosthesis

\section{Introduction}

Total laryngectomy and pharyngolaryngectomy is indicated in the treatment of locally advanced squamous cell carcinoma of the larynx and hypopharynx, or as a salvage procedure. However, it is associated with major morbidity, particularly the loss of normal voice, and the subsequent psychological and functional impact. ${ }^{1-3}$ Consequently, functional voice rehabilitation is becoming increasingly important for improving patients' quality of life. Tracheoesophageal voice via a shunt valve is currently regarded as the 'gold standard' for voice rehabilitation. ${ }^{4,5}$

The advantages of tracheoesophageal speech valves over oesophageal speech include: immediate voice, high success rates and the ability to sustain speech with a more fluent quality than that achieved with oesophageal speech. ${ }^{4,5}$

It is essential for the valve to have a good fit as this ensures both a water- and air-tight seal, thereby maximising voice quality and reducing the risk of aspiration and valve displacement. Leakage, both through the centre of the valve and around the periphery, is a common problem, occurring in up to 27 per cent of patients. ${ }^{6}$ On average, speech valves need to be replaced every three to four months. ${ }^{7,8}$ The replacement procedure is uncomfortable for the patient. It also increases the risk of: scar tissue formation around the stoma, stoma stenosis and a dysfunctional tracheoesophageal puncture. ${ }^{9}$ In some cases, this may require recreation of the fistula under general anaesthesia.

Re-insertion of the valve becomes increasingly difficult in patients who have had numerous fistulas created, when there is alteration to the neopharyngeal anatomy or when the patient is not fit for further surgical procedures because of medical co-morbidites. ${ }^{10}$

Here we discuss a novel method of speech valve insertion in a patient in whom traditional techniques had failed and placement under general anaesthesia was contraindicated.

\section{Case report}

A 69-year-old male underwent salvage total laryngectomy for recurrent squamous cell carcinoma of the larynx in 2003 , having previously been treated with radical radiotherapy. He had a primary tracheoesophageal fistula created at the time of his laryngectomy. The patient underwent recreation of the fistula in 2011 and again in 2014 following valve extrusion.

The patient had initially been seen in the clinic because of ongoing leakage from around the periphery of his valve. Further tracheoesophageal puncture attempts were contraindicated because of the significant scarring around the site. Placement under general anaesthesia was not possible because of his medical co-morbidities. He was known to have a tight neopharyngeal stricture below the level of the tracheoesophageal puncture that had been dilated on several occasions.

The valve had been removed in clinic, but numerous attempts to replace it using both Blom-Singer ${ }^{\circledR}$ and Provox ${ }^{\circledR}$ valve systems had been unsuccessful. The size of the puncture was adequate; the failure was instead thought to be a result of the angle of the puncture caused by the stricture just below it.

We performed a novel method of speech valve insertion to overcome the aforementioned issues. The patient was positioned sitting up. The nasopharynx, oral cavity and stoma were prepared with lidocaine hydrochloride 5 per cent and phenylephrine hydrochloride 0.5 per cent topical solution. The metallic guide wire was removed from an $8 \mathrm{Fr}$ gauge fine bore nasogastric (NG) tube and disposed of. The guide wire from the Provox primary puncture set was introduced into the lumen of the NG tube, in place of the metal guide wire. The NG tube was inserted through the tracheoesophageal puncture and directed upwards. The NG tube tip protected the posterior pharyngeal wall from the sharper tip of the Provox guide wire. Once the tip of the NG was 
visible within the oral cavity, it was grasped using a pair of straight artery clips and pulled through. The Provox guide wire was controlled intra-orally and the NG tube was removed through the tracheoesophageal puncture.

The Provox valve was mounted onto the introducer using the standard method. It was then mounted onto the guide wire intra-orally, and railroaded down the guidewire and through the tracheoesophageal puncture. The guide wire was removed through the tracheoesophageal puncture and the valve was trimmed as normal.

The patient was observed for 1 hour and then given a trial of sterile water to check for leakage around the valve. He was discharged several hours later and was able to tolerate a normal diet.

The patient reported minimal discomfort from the procedure and could vocalise immediately after insertion of the valve.

\section{Discussion}

The described technique represents an alternative method for replacing speech valves in patients where the standard methods have failed, in our case because of a difficult tracheoesophageal puncture angle secondary to altered anatomy, or when placement under general anaesthesia is contraindicated.

The method is relatively quick and causes little trauma to the surrounding tissues. It is particularly useful as it avoids the need for a general anaesthetic. It is cost-effective, as it does not require any equipment other than that which is available in most ENT wards and operating theatres. However, patient selection is key, as patient compliance with the procedure is essential to its success.

\section{References}

1 Bozec A, Poissonnet G, Chamorey E, Casanova C, Vallicioni J, Demard $\mathrm{F}$ et al. Free-flap head and neck reconstruction and quality of life: a 2-year prospective study. Laryngoscope 2008; 118:874-80

2 Dassonville O, Poissonnet G, Chamorey E, Vallicioni J, Demard F, Santini J et al. Head and neck reconstruction with free flaps: a report on 213 cases. Eur Arch Otorhinolaryngol 2008;265: 85-95

3 Robertson SM, Yeo JC, Dunnet C, Young D, Mackenzie K. Voice, swallowing, and quality of life after total laryngectomy: results of the west of Scotland laryngectomy audit. Head Neck 2012;34:59-65

4 de Casso C, Slevin NJ, Homer JJ. The impact of radiotherapy on swallowing and speech in patients who undergo total laryngectomy. Otolaryngol Head Neck Surg 2008;139:792-7

5 Emerick KS, Tomycz L, Bradford CR, Lyden TH, Chepeha DB, Wolf GT et al. Primary versus secondary tracheoesophageal puncture in salvage total laryngectomy following chemoradiation. Otolaryngol Head Neck Surg 2009;140:386-90

6 Laccourreye O, Menard M, Crevier-Buchman L, Couloigner V, Brasnu D. In situ lifetime, causes for replacement, and complications of the Provox voice prosthesis. Laryngoscope 1997;107: $527-30$

7 Op de Coul BM, Hilgers FJ, Balm AJ, Tan IB, van den Hoogen FJ, van Tinteren $\mathrm{H}$. A decade of postlaryngectomy vocal rehabilitation in 318 patients: a single institution's experience with consistent application of provox indwelling voice prostheses. Arch Otolaryngol Head Neck Surg 2000;126:1320-8

8 Brown DH, Hilgers FJ, Irish JC, Balm AJ. Postlaryngectomy voice rehabilitation: state of the art at the millennium. World $J$ Surg 2003;27:824-31

9 Free RH, Van der Mei HC, Dijk F, Van Weissenbruch R, Busscher HJ, Albers FW. Biofilm formation on voice prostheses: influence of dairy products in vitro. Acta Otolaryngol 2000;120:92-9

10 Malik T, Bruce I, Cherry J. Surgical complications of tracheaoesophageal puncture and speech valves. Curr Opin Otolaryngol Head Neck Surg 2007;15:117-22

Address for correspondence:

Mr C P Yiannakis,

Department of Otolaryngology,

Monklands District General Hospital,

Monkscourt Ave,

Airdrie ML6 0JS

Scotland, UK

E-mail: c.p.yiannakis@doctors.org.uk

Mr C P Yiannakis takes responsibility for the integrity of the content of the paper

Competing interests: None declared 